Journal of the Operations Research

Society of Japan

Vol. 41, No. 1, March 1998

\title{
WORST-CASE PERFORMANCE OF ATM MULTIPLEXER WITH GCRA-CONFORMING ARRIVAL PROCESSES
}

\author{
Shigeo Shioda \\ $N T T$
}

(Received March 3, 1997; Revised October 7, 1997)

\begin{abstract}
The worst-case performance of an ATM multiplexer, in which each multiplexed connection sends cells so as to conform to a generic cell-rate algorithm (GCRA), has been examined. Specifically, the concept of large-deviation ordering is introduced to find the "worst" cell-arrival process, i.e., the one yielding the worst performance of the ATM multiplexer. Several interesting results were obtained. First, the cell-loss ratio (CLR) of a bufferless model does not always give the worst CLR when the buffer size per connection is less than $c / M$, where $c$ is the capacity of the outgoing link per connection, and $M$ is the peak rate of the connection. Second, the cell-delay variation tolerance does not affect the worst-case performance if the buffer size is sufficiently large. Finally, theoretical support was derived for Yamanaka's conjecture that the greedy on-off pattern is not always the worst cell-arrival pattern among those conforming to the dual GCRAs.
\end{abstract}

\section{Introduction}

The broadband integrated services digital network is to be based on the asynchronous transfer mode (ATM), whereby information is transmitted in the form of fixed-sized packets called ATM cells. Users of constant or variable bit-rate connections share the switching and transmission facilities by coding and segmenting their information into cell payloads and transmitting them as cell streams; these streams are multiplexed, switched, and routed through the ATM network. The high transmission speed of ATM makes propagation delays consequential, making it difficult to design an effective reactive control mechanism. In ATM networks, therefore, preventive traffic controls are mainly used to keep the quality of service at a satisfactory level.

We will first briefly summarize the preventive traffic controls in ATM networks. At connection setup, the user specifies both the quality-of-service (QoS) requirements and, using a source traffic descriptor; the anticipated traffic characteristics of the connection. Network resources for the connection are then assigned on the basis of the source traffic descriptor values and the QoS requirements. If there are not enough resources available, the connection is rejected. If the connection is accepted, the actual traffic characteristics are checked for conformity with the values specified at connection setup by monitoring the cell stream. If the characteristics do not conform, a penalty is imposed on the connection, e.g., some cells from the connection may be discarded. The first of these procedures, at connection setup, is called connection admission control, and the procedure after connection setup is called usage parameter control (UPC).

A source traffic descriptor is a set of traffic parameters, including the peak cell rate (PCR), the sustainable cell rate (SCR), the cell-delay variation tolerance (CDVT), and the burst tolerance (BT). Conformance to these parameters is algorithmically defined in terms 
of a generic cell-rate algorithm (GCRA) [1], which is a kind of leaky bucket algorithm. In this algorithm, whether each cell conforms to specified peak cell rate $M$ and to cell-delay variation tolerance $T_{c}$ is determined as follows [14]. Let $\left\{a_{k}\right\}$ denote the set of observed cellarrival times and $\left\{c_{k}\right\}$ denote a set of theoretical cell-arrival times; $y_{k}\left(=c_{k}-a_{k}\right)$ represents the variation in cell delay affecting the $k$ th cell. A cell conforms to the pair $\left(M, T_{c}\right)$ if and only if $y_{k} \leq T_{c}$, when the sequence $\left\{c_{k}\right\}$ is computed as follows:

$$
\begin{aligned}
& c_{k+1}=a_{k}+1 / M \quad y_{k}<0, \\
& c_{k+1}=c_{k}+1 / M \quad 0 \leq y_{k}<T_{c}, \\
& c_{k+1}=c_{k} \quad T_{c} \leq y_{k} .
\end{aligned}
$$

The GCRA specified by the pair $\left(M, T_{c}\right)$ physically corresponds to the leaky bucket algorithm whose leak rate is $M$ and bucket size is $\left\lfloor M T_{c}\right\rfloor$ (Fig. 1). The conformance to a specified SCR and BT is defined in the same manner.

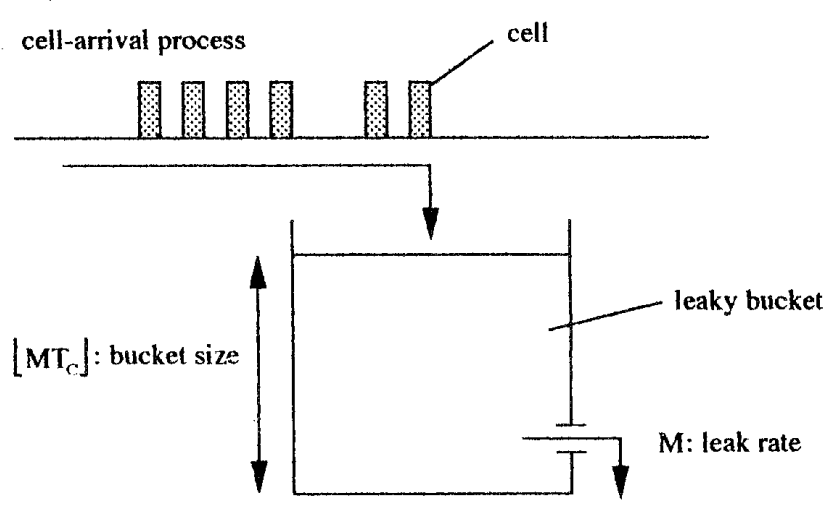

Fig. 1. GCRA parameters and leak bucket.

As well as defining conformance, the GCRA is used in the UPC function, which sits on the network side of the user network interface before the first ATM switching or multiplexing function in the network. There is a wide variety of traffic patterns that conform to the source traffic descriptor, and the network needs to accept all of these patterns without violating the QoS guarantees. Thus, to design traffic-control and traffic-management procedures, we need to know the worst-case traffic among those conforming to the GCRA. The general belief is that greedy on-off patterns, which cyclically send cells at full speed until the bucket is full and then go silent until it leaks empty again (Fig. 2), are the worst case. This conjecture is intuitive but unproved. Yamanaka et al. [23] reported a counterexample based on simulation; they claim another class of sources (depicted in Fig. 3) represents even worse traffic. However, they considered only a single conforming source, not multiplexed sources. Doshi [8] also investigated this problem and suggested that the worst-case pattern may depend on the source mix (heterogeneous or homogeneous), the characteristics of the multiplexer (buffered or unbuffered), etc. Mitra et al. [11] considered the resource allocation strategy for the ATM network, assuming that the greedy on-off pattern is the worst pattern without conclusive proof. Among cell-arrival patterns conforming to the GCRA, Lee [17] found the pattern that maximizes the average waiting time. However, more important QoS factors, for example, the cell-loss ratio (CLR) or $x$-percentile delay, were not discussed. Shioda et al. [20] discussed the worst-case performance of a multiplexer for connections conforming to a pair of PCR and CDVT and derived the upper bound of the 
CLR. Tsuchiya et al. [22] extended Shioda's result to derive the upper bound of the CLR when the connections conforming to the PCR, SCR, and BT are multiplexed. This upper bound, which was derived in the long-range-correlation limit, overestimates the actual CLR because it essentially neglects the cell-buffering effect.

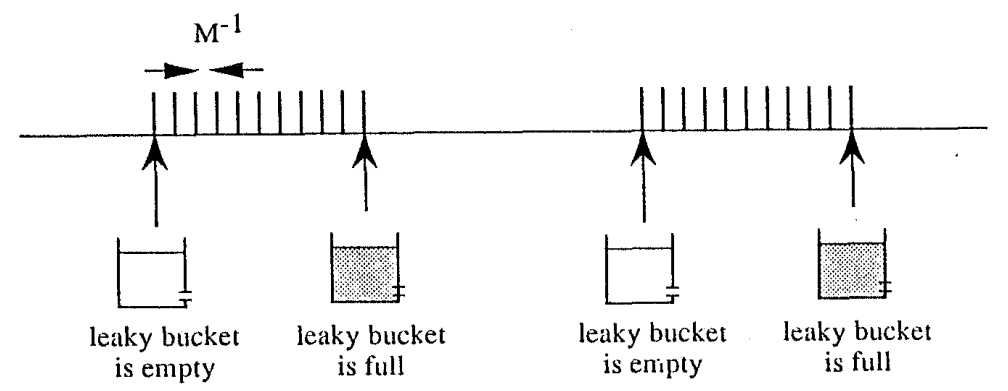

Fig. 2. Greedy on-off pattern.

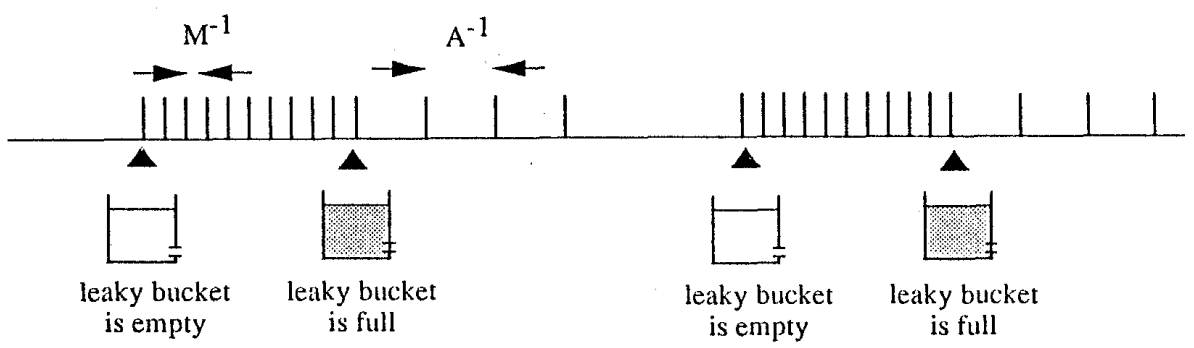

Fig. 3. Yamanaka's conjecture: worst cell-arrival pattern.

In this paper, we provide a theoretical framework for finding the worst-case pattern by using large-deviation analysis. One important measure of QoS is the CLR, which should usually be very small, typically of the order of $10^{-6}$ to $10^{-10}$. Large-deviation analysis is a very powerful tool for handling such a rare event as cell loss in this situation, and the application of large-deviation techniques to ATM traffic control has been extensively studied $[4,5,9,10,12,13,16,24]$.

In particular, large-deviation analysis explains well the asymptotics of queue-length distributions in the regime of large buffers. The asymptotics in the large-buffer regime are, however, of no use in analyzing the performance of the multiplexer under the GCRA-conforming arrival process because the number of cells arriving from such a process is always bounded from above, and the queue length is also bounded. Instead, in the case where the $L$-fold superposition of GCRA-conforming connections is multiplexed, we analyze the asymptotic behavior of queue-length distributions as $L \rightarrow \infty$. This is reasonable because the number of connections multiplexed on an outgoing link of a multiplexer may be of the order $10^{2}-10^{3}$ due to the large capacity of the outgoing link (150-600 Mbps).

Let $Q^{L}$ be the number of cells (more precisely, workload) in the buffer at an ATM multiplexer shared by $L$ identical independent connections (Fig. 4 ). The buffer is served at constant rate $L c$, and the buffer size is $L \kappa$. As shown in Sec. 2, the large $L$ asymptotic for the queue-length distribution yields

$$
\lim _{L \rightarrow \infty} \frac{1}{L} \log \mathbf{P}\left[Q^{L} \geq L \kappa\right]=-\inf _{t>0} I_{t}(\kappa+c t),
$$


or less formally we have

$$
C L R \simeq \beta \exp \left\{-L \inf _{t>0} I_{t}(\kappa+c t)\right\} .
$$

Here, $I_{t}(\cdot)$ is the rate funciton of an individual source defined by

$$
I_{t}(x)=\sup _{\theta}\left[\theta x-\log E\left[e^{\theta A_{t}}\right]\right]
$$

where $A_{t}$ is the number of cells arriving during $[0, t)$. Equation (1.2) implies that the rate function can be used to compare of cell-arrival processes. That is, cell-arrival process $\varphi$ can be said to be worse than $\phi$ when

$$
\inf _{t>0} I_{t}(\kappa+c t \mid \varphi) \leq \inf _{t>0} I_{t}(\kappa+c t \mid \phi)
$$

where $I_{t}(\cdot \mid \varphi)$ denotes the rate function of cell-arrival process $\varphi$. We call this ordering criterion large-deviation ordering. It gives the ordering of the system performance in the limit of large $L$ when the system is scaled using $L$. (A similar concept was introduced in Ref. [3] for the discussion of negligible CDV.) In this paper we focus on finding the worst cell-arrival process in the sense of large-deviation ordering.

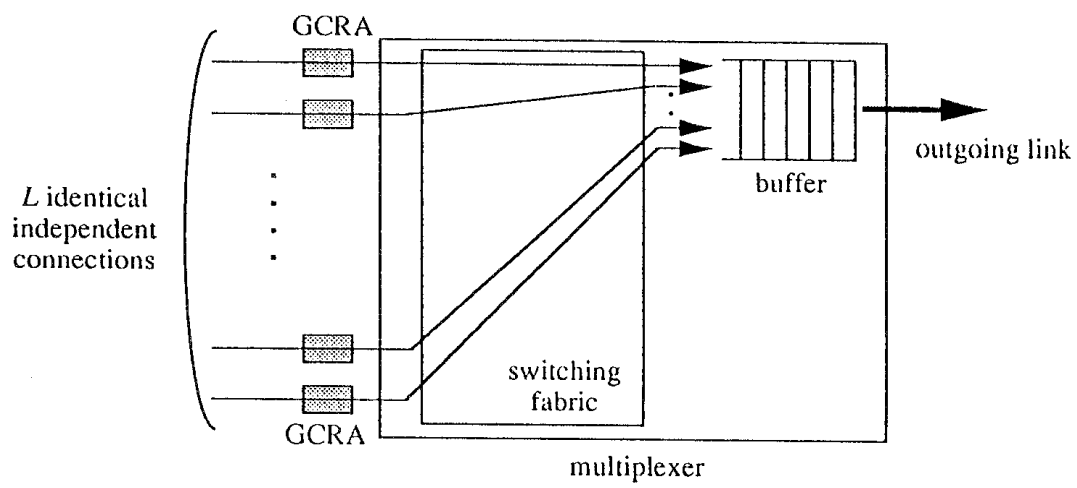

Fig. 4. ATM multiplexer.

This paper is organized as follows. We begin in Sec. 2 by briefly summarizing the large $L$ (number of multiplexed connections) asymptotics of queue-length distributions. Then, based on these asymptotics, we introduce the concept of large-deviation ordering for comparing cell-arrival processes. The worst arrival process in the sense of large-deviation ordering is also derived for "bounded arrival processes", which are very general point processes, including GCRA-conforming arrival processes. In Sec. 3, we use the results obtained in Sec. 2 to derive the worst arrival process among all GCRA-conforming arrival processes. These results are used in Sec. 4 to discuss the worst-case performance of a multiplexer in three cases: (i) $C D V T=0$ and $B T=\infty$, (ii) $C D V T>0$ and $B T=\infty$, and (iii) $C D V T=0$ and $B T<\infty$. We show a few numerical examples in Sec. 5 to numerically illustrate the results derived in Sec. 4. Finally, we conclude in Sec. 6 with a discussion of some unsolved problems.

\section{Worst bounded cell-arrival process}

\subsection{Large $L$ asymptotics of cell-loss ratio}

As a preliminary, we will briefly summarize the CLR asymptotics in the regime of a large number of multiplexed connections [4]. Assume that $L$ identical independent cellarrival processes share a multiplexer. The multiplexer is served by a fixed-rate channel with 
a capacity of $L c$ cells in unit time. Cells that cannot be immediately transmitted on the outgoing channel are queued in a buffer of size $L \kappa$. Excess cells arriving at a full buffer are considered lost.

Let $Q^{L}$ denote the workload remaining in the multiplexer at $t=0$. (Here, the workload is normalized by the service time of one cell. Thus, the integer part of $Q^{L}$ can be considered to be the number of cells in the buffer.) By the Lindley equation, we have [4]

$$
Q^{L}=\sup _{t>0}\left\{A_{t}^{L}-c L t\right\}
$$

where $A_{t}^{L}$ is the number of cells arriving during $[-t, 0)$ from $L$ cell arrival processes. It was shown in Ref. [4] that if point process $A_{t}$ satisfies the large-deviation principle,

$$
\begin{aligned}
\lim _{L \rightarrow \infty} L^{-1} \log \mathbf{P}\left[Q^{L} \geq L \kappa\right] & =\lim _{L \rightarrow \infty} L^{-1} \log \mathbf{P}\left[\sup _{t>0}\left(A_{t}^{L}-c L t\right) \geq L \kappa\right] \\
& =\sup _{t>0} \lim _{L \rightarrow \infty} L^{-1} \log \mathbf{P}\left[\frac{A_{t}^{L}}{L} \geq \kappa+c t\right]=-\inf _{t>0} I_{t}(\kappa+c t),
\end{aligned}
$$

where

$$
I_{t}(\kappa+c t) \stackrel{\text { def }}{=} \sup _{\theta}\left\{\theta(\kappa+c t)-\log \mathbf{E}\left[e^{\theta A_{t}}\right]\right\}
$$

The function $I_{t}(x)$ is considered to be a time-dependent rate function of the individual-cellarrival process. Thus, we approximately have

$$
C L R \simeq \mathbf{P}\left[Q^{L} \geq \kappa L\right] \simeq \beta \exp \left\{-L \inf _{t>0} I_{t}(\kappa+c t)\right\}
$$

Remark: It is easy to see that a stronger limit than Eq. (2.2) holds. Let $\Lambda_{t}(\theta)=\log \mathbf{E}\left[e^{\theta A_{t}}\right]$, and $\theta_{t}^{*}$ be the solution of the equation $\kappa+c t=\Lambda_{t}^{\prime}\left(\theta_{t}^{*}\right)$. We first note that the Bahadur and Rao theorem ([7] Theorem 3.7.4, [15]) gives for $t>0$

$$
\mathbf{P}\left[A_{t}^{L}-c L t \geq L \kappa\right] \sim \frac{1}{\theta_{t}^{*} \sqrt{2 \pi L \Lambda_{t}^{\prime \prime}\left(\theta_{t}^{*}\right)}} \exp \left\{-L I_{t}(\kappa+c t)\right\}
$$

where $f(L) \sim g(L)$ means that $f(L) / g(L) \rightarrow 1$ as $L \rightarrow \infty$. Because $t$ is arbitrary, we have

$$
\begin{aligned}
\mathbf{P}\left[Q^{L} \geq L \kappa\right] & =\mathbf{P}\left[\sup _{t>0}\left\{A_{t}^{L}-c L t\right\} \geq L \kappa\right] \geq \sup _{t>0} \mathbf{P}\left[A_{t}^{L}-c L t \geq L \kappa\right] \\
& \sim \sup _{t>0}\left\{\frac{1}{\theta_{t}^{*} \sqrt{2 \pi L \Lambda_{t}^{\prime \prime}\left(\theta_{t}^{*}\right)}} \exp \left\{-L I_{t}(\kappa+c t)\right\}\right\} .
\end{aligned}
$$

Thus, we obtain the following lower bound for the large $L$ asymptotics of the workload distribution:

$$
\mathbf{P}\left[Q^{L} \geq L \kappa\right] \geq \frac{1}{\theta_{\tau}^{*} \sqrt{2 \pi L \Lambda_{\tau}^{\prime \prime}\left(\theta_{\tau}^{*}\right)}} \exp \left\{-L \inf _{t>0} I_{t}(\kappa+c t)\right\},
$$

where $f(L) \geq g(L)$ means that $f(L) / g(L) \rightarrow \varepsilon \geq 1$ as $L \rightarrow \infty$, and $\tau$ attains the infimum $\inf _{t>0} I_{t}(\kappa+c t)$. 


\subsection{Large-deviation ordering}

The cell-loss ration asymptotics (2.4) naturally suggest the notion of large-deviation ordering, which is defined as follows.

Definition 2.1 Let $\varphi$ and $\phi$ be two cell-arrival processes. We say that $\varphi$ is worse than $\phi$ in the sense of large-deviation ordering if

$$
\inf _{t>0} I_{t}(\kappa+c t \mid \varphi) \leq \inf _{t>0} I_{t}(\kappa+c t \mid \phi)
$$

where $I_{t}(\cdot \mid \phi)$ denotes the rate function of cell-arrival process $\phi$. This is denoted as $\varphi \leq_{L D} \phi$. In the following, we investigate the worst arrival pattern in the sense of large-deviation ordering among all "bounded" cell-arrival inputs. Here, "bounded" means that the number of cells arriving during an interval of length $t$ is essentially bounded by some positive value $m_{t}$ with probability 1 . Let $\Omega(\mathcal{A})$ denote the set of all stationary and ergodic cell-arrival processes satisfying given constraint $\mathcal{A}$. The worst arrival process under $\mathcal{A}$-worst in the sense of large-deviation ordering-is defined as follows:

Definition 2.2 Cell-arrival process $\varphi \in \Omega(\mathcal{A})$ is said to be the worst in the sense of large deviation ordering if $\varphi \leq_{L D} \phi$ for every $\phi \in \Omega(\mathcal{A})$.

In the following, we specifically consider the worst cell-arrival process under the following "bounded" constraint:

$$
\mathcal{A}=\left\{\mathbf{M}\left[A_{t}\right] \leq m_{t}, \mathbf{E}\left[A_{t}\right] \leq a_{t}, t \in[0, \infty)\right\}
$$

where

$$
\mathbf{M}\left[A_{t}\right] \stackrel{\text { def }}{=} \max _{k}\left\{k \mid \mathbf{P}\left[A_{t}=k\right]>0\right\}
$$

Lemma 2.1 If cell arrival process $\phi$ satisfies the constraint $\mathcal{A}$ given by Eq. (2.9), then

$$
\mathbf{E}\left[e^{\theta A_{t}^{\phi}}\right] \leq \frac{a_{t}}{m_{t}} e^{\theta m_{t}}+\left(1-\frac{a_{t}}{m_{t}}\right),
$$

where $A_{t}^{\phi}$ denotes the number of cells arriving during $[-t, 0)$ from cell-arrival process $\phi$.

Proof Let $P_{t}^{\phi}(k)$ and $p_{t}^{\phi}(k)$ be the distribution and density functions of $A_{t}^{\phi}$, respectively. Consider an associated cell-arrival process, $\psi$, whose distribution and density functions, $P_{t}^{\psi}(k)$ and $p_{t}^{\psi}(k)$, are given by

$$
P_{t}^{\psi}(k)=\left\{\begin{array}{ll}
1-a_{t}^{\phi} / m_{t}^{\phi} & k<m_{t}^{\phi}, \\
1 & k=m_{t}^{\phi},
\end{array} \quad \text { and } \quad p_{t}^{\psi}(k)= \begin{cases}a_{t}^{\phi} / m_{t}^{\phi} & k=m_{t}^{\phi} \\
1-a_{t}^{\phi} / m_{t}^{\phi} & k=0 \\
0 & \text { otherwise }\end{cases}\right.
$$

where

$$
a_{t}^{\phi}=\sum_{k=0}^{\infty} k p_{t}^{\phi}(k), \quad \text { and } \quad m_{t}^{\phi}=\max \left\{k \mid p_{t}^{\phi}(k)>0\right\}
$$

We define

$$
k_{c u t}^{\phi}=\min \left\{k \mid P_{t}^{\phi}(k) \geq 1-a_{t}^{\phi} / m_{t}^{\phi}\right\} .
$$

Observe that $A_{t}^{\phi}$ and $A_{t}^{\psi}$ satisfy the cut criterion of distribution [21] because

$$
P_{t}^{\phi}(k) \leq P_{t}^{\psi}(k) \quad k<k_{c u t}^{\phi}, \quad \text { and } \quad P_{t}^{\phi}(k) \geq P_{t}^{\psi}(k) \quad k>k_{\text {cut }}^{\phi} .
$$


It was shown ([21] Proposition 1.5.1) that random variables $X$ and $Y$ satisfying the cut criterion of distribution have $X \leq_{c x} Y$, where $\leq_{c x}$ denotes convex ordering. Hence, $A_{t}^{\phi} \leq_{c x}$ $A_{t}^{\psi}$, and thus

$$
\mathbf{E}\left[e^{\theta A_{t}^{\phi}}\right] \leq \mathbf{E}\left[e^{\theta A_{t}^{\psi}}\right]=\frac{a_{t}^{\phi}}{m_{t}^{\phi}} e^{\theta m_{t}^{\phi}}+\left(1-\frac{a_{t}^{\phi}}{m_{t}^{\phi}}\right),
$$

where we use the fact that $e^{x}$ is an increasing convex function. Because the final expression of Eq. (2.16) is increasing for $a_{t}^{\phi}$ and $m_{t}^{\phi}$, it follows from the definition of $\Omega(\mathcal{A})$ that

$$
\mathbf{E}\left[e^{\theta A_{t}^{\phi}}\right] \leq \frac{a_{t}}{m_{t}} e^{\theta m_{t}}+\left(1-\frac{a_{t}}{m_{t}}\right)
$$

This completes the proof.

The following statement is the consequence of Lemma 2.1 .

Theorem 2.1 If cell arrival process $\phi$ satisfies the constraint $\mathcal{A}$ given by Eq. (2.9), then

$$
I_{t}(x \mid \phi) \geq \hat{I}\left(x \mid m_{t}, a_{t}\right)
$$

where

$$
\hat{I}\left(x \mid m_{t}, a_{t}\right) \stackrel{\text { def }}{=} \frac{x}{m_{t}} \log \frac{x}{a_{t}}+\left(1-\frac{x}{m_{t}}\right) \log \frac{1-x / m_{t}}{1-a_{t} / m_{t}}
$$

Proof Substituting Eq. (2.11) into Eq. (2.3), we have

$$
\begin{aligned}
I_{t}(x \mid \phi) & \geq \sup _{\theta}\left[\theta x-\log \left\{\frac{a_{t}}{m_{t}} e^{\theta m_{t}}+\left(1-\frac{a_{t}}{m_{t}}\right)\right\}\right] \\
& =\frac{x}{m_{t}} \log \frac{x}{a_{t}}+\left(1-\frac{x}{m_{t}}\right) \log \frac{1-x / m_{t}}{1-a_{t} / m_{t}} .
\end{aligned}
$$

This completes the proof.

Let $\Psi_{T} \in \Omega(\mathcal{A})$ be the cell-arrival process whose density function $p_{t}^{\Psi_{T}}(k)$ satisfies the following at $t=T$ :

$$
p_{T}^{\Psi_{T}}(k)= \begin{cases}a_{T} / m_{T} & k=m_{T} \\ 1-a_{T} / m_{T} & k=0 \\ 0 & \text { otherwise }\end{cases}
$$

Corollary 2.1 Cell-arrival process $\Psi_{T}$ is the worst in $\Omega(\mathcal{A})$ in the sense of large-deviation ordering if for any $t$,

$$
\hat{I}\left(\kappa+c T \mid m_{T}, a_{T}\right) \leq \hat{I}\left(\kappa+c t \mid m_{t}, a_{t}\right)
$$

Proof It is easy to see that $I_{T}\left(\kappa+c T \mid \Psi_{T}\right)=\hat{I}\left(\kappa+c T \mid m_{T}, a_{T}\right)$. Thus, for all $\phi \in \Omega(\mathcal{A})$,

$$
\begin{aligned}
\inf _{t>0} I_{t}(\kappa+c t \mid \phi) & \geq \inf _{t>0} \hat{I}\left(\kappa+c t \mid m_{t}, a_{t}\right)=\hat{I}\left(\kappa+c T \mid m_{T}, a_{T}\right)=I_{T}\left(\kappa+c T \mid \Psi_{T}\right) \\
& \geq \inf _{t>0} I_{t}\left(\kappa+c t \mid \Psi_{T}\right)
\end{aligned}
$$

and therefore $\Psi_{T} \leq_{L D} \phi$. 


\section{Worst case performance with GCRA-conforming arrival processes: general framework}

Here, we examine the cell arrival processes that conform to the set of four traffic parameters: peak cell rate $M$, cell-delay variation tolerance $T_{c}$, sustainable cell rate $A$, and burst tolerance $T_{b}$. The conformance definitions of these four parameters are given in terms of the GCRA, as explained in Sec. 1. The cell-arrival processes conforming to these traffic parameters are bounded cell-arrival processes and satisfy the following constraint:

$$
\begin{aligned}
& \mathcal{A}_{G C R A}=\left\{\mathbf{M}\left[A_{t}\right] \leq m_{t}^{G C R A}, \quad \mathbf{E}\left[A_{t}\right] \leq A t, \quad t \in[0, \infty)\right\}, \\
& m_{t}^{G C R A} \stackrel{\text { def }}{=}\left\{1+\left\lfloor\left(t+T_{c}\right) M\right\rfloor\right\} \wedge\left\{1+\left\lfloor\left(t+T_{b}\right) A\right\rfloor\right\},
\end{aligned}
$$

where $a \wedge b=\min \{a, b\}$. Conforming to these four parameters is equivalent to conforming to dual GCRAs: the first-stage GCRA is specified by the pair $\left(M, T_{c}\right)$, and the second-stage GCRA is specified by the pair $\left(A, T_{b}\right)$ (Fig. 5). It was shown in Ref. [20] that the number of cells arriving during a period of length $t$ from a cell-arrival process conforming to the pair $\left(M, T_{c}\right)$ is bounded by $1+\left\lfloor\left(t+T_{c}\right) M\right\rfloor$. Similarly, the number of cells arriving during a period of length $t$ from the cell-arrival process conforming to the pair $\left(A, T_{b}\right)$ is bounded by $1+\left\lfloor\left(t+T_{b}\right) A\right\rfloor$. Thus,

$$
\mathbf{M}\left[A_{t}\right] \leq\left\{1+\left\lfloor\left(t+T_{c}\right) M\right\rfloor\right\} \wedge\left\{1+\left\lfloor\left(t+T_{b}\right) A\right\rfloor\right\} .
$$

Another condition, $\mathbf{E}\left[A_{t}\right] \leq A t$, is the direct consequence of the definition of the sustainable cell rate (the SCR is the upper bound of the average cell rate).

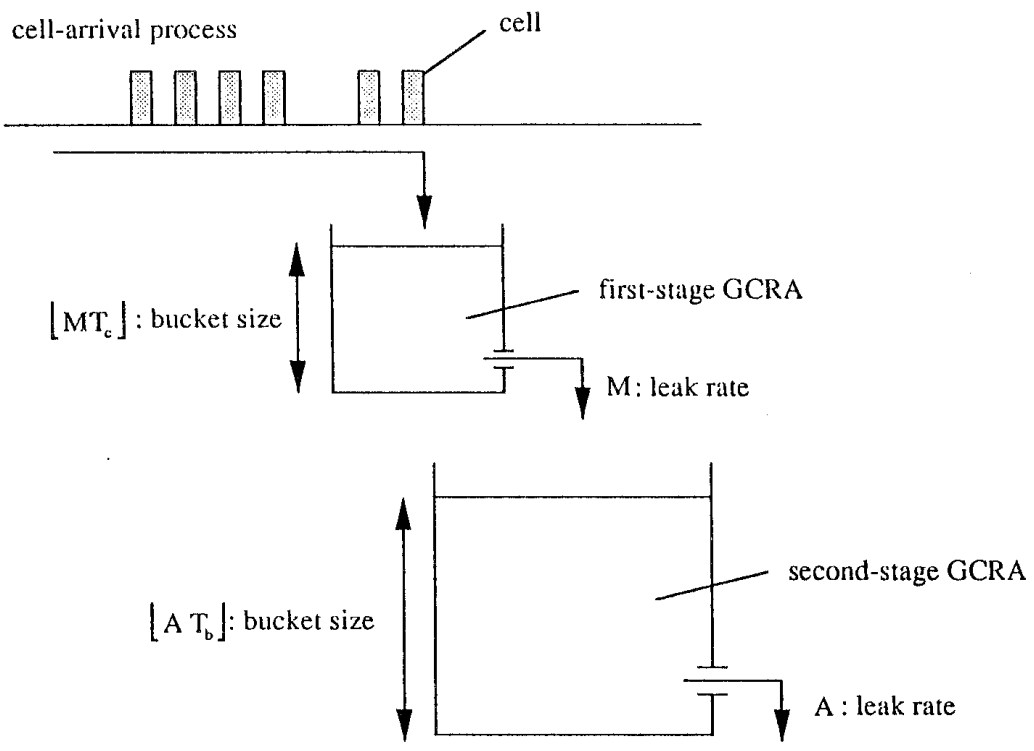

Fig. 5. Dual GCRAs.

Let $\Psi_{T}^{G C R A}$ be the cell-arrival process whose density function $p_{t}^{\Psi_{T}^{G C R A}}(k)$ satisfies the following at $t=T$ :

$$
p_{T}^{\Psi_{T}^{G C R A}}= \begin{cases}A T / m_{T}^{G C R A} & k=m_{T}^{G C R A} \\ 1-A T / m_{T}^{G C R A} & k=0 \\ 0 & \text { otherwise }\end{cases}
$$


Lemma 3.1 If cell-arrival process $\phi$ satisfies the constraint $\mathcal{A}_{G C R A}$, then

$$
I_{T}(\kappa+c T \mid \phi) \geq I_{T}\left(\kappa+c T \mid \phi_{T}^{G C R A}\right) \geq f\left(p_{T}, q_{T}\right)
$$

where

$$
f(\alpha, \beta) \stackrel{\text { def }}{=} \beta \log \frac{\beta}{\alpha}+(1-\beta) \log \frac{1-\beta}{1-\alpha}, \quad q_{T}=\frac{\kappa+c T}{m_{T}^{G C R A}}, \quad p_{T}=\frac{A T}{m_{T}^{G C R A}} .
$$

Proof It follows from Theorem 2.1 that for every $\phi \in \Omega\left(\mathcal{A}_{G C R A}\right)$

$$
I_{T}(\kappa+c T \mid \phi) \geq \hat{I}\left(\kappa+c T \mid m_{T}^{G C R A}, A T\right)=f\left(p_{T}, q_{T}\right) .
$$

It is easy to see that $I\left(\kappa+c T \mid \Psi_{T}^{G C R A}\right)=f\left(p_{T}, q_{T}\right)$.

The following is the GCRA-conforming arrival-process version of Corollary 2.1.

Lemma 3.2 Cell-arrival process $\Psi_{T}^{G C R A}$ is the worst in $\Omega\left(\mathcal{A}_{G C R A}\right)$ in the sense of largedeviation ordering if $f\left(p_{T}, q_{T}\right) \leq f\left(p_{t}, q_{t}\right)$ for any $t$.

Proof For any $\phi \in \Omega\left(\mathcal{A}_{G C R A}\right)$,

$$
\begin{aligned}
\inf _{t>0} I_{t}(\kappa+c t \mid \phi) & \geq \inf _{t>0} f\left(p_{t}, q_{t}\right)=f\left(p_{T}, q_{T}\right)=I_{T}\left(\kappa+c T \mid \Psi_{T}^{G C R A}\right) \\
& \geq \inf _{t>0} I_{t}\left(\kappa+c t \mid \Psi_{T}^{G C R A}\right) .
\end{aligned}
$$

Thus, $\Psi_{T}^{G C R A} \leq_{L D} \phi$.

Note that the stability condition of the queue requires $c<A$, and therefore $q_{t}>p_{t}$. Also note that no cells are lost when $q_{t} \geq 1$ because $q_{t} \geq 1$ implies $\kappa+c t \geq m_{t}^{G C R A}$. Thus, $f\left(p_{t}, q_{t}\right)$ should be infinite for $q_{t} \geq 1$ due to the physical meaning of the rate function. The following lemma will be used in the rest of this paper.

Lemma 3.3 If $\alpha \leq \beta \leq 1$, then $f(\alpha, \beta)$ is an increasing function of $\beta$ and a decreasing function of $\alpha$.

Proof: Observe that

$$
\frac{\partial}{\partial \alpha} f(\alpha, \beta)=-\frac{\beta-\alpha}{\alpha(1-\alpha)} \leq 0, \quad \frac{\partial}{\partial \beta} f(\alpha, \beta)=\log \frac{\beta(1-\alpha)}{\alpha(1-\beta)} \geq \log 1=0
$$

which completes the proof.

Lemma 3.2 implies that the worst cell-arrival process exists in the uncountable set $\left\{\Psi_{t}^{G C R A}\right\}$. In the following, to simplify the discussion, we focus on finding the worst arrival process in the countable set $\left\{\Psi_{t_{n}}^{G C R A}\right\}_{n=1}^{\infty}\left(\subset\left\{\Psi_{t}^{G C R A}\right\}\right)$, where $t_{1}, t_{2}, \cdots$ (Fig. 6) are defined by

$$
t_{n} \stackrel{\text { def }}{=} \inf \left\{t \mid m_{t}^{G C R A}=n\right\}=\left\{\frac{n-1}{M}-T_{c}\right\} \vee\left\{\frac{n-1}{A}-T_{b}\right\} \vee 0,
$$

where $a \vee b=\max \{a, b\}$. In this setting, $\Psi_{N}^{G C R A} \stackrel{\text { def }}{=} \Psi_{t_{N}}^{G C R A}$ is the worst in $\Omega\left(\mathcal{A}_{G C R A}\right)$ in the sense of large-deviation ordering if

$$
f\left(p_{N}, q_{N}\right) \leq f\left(p_{n} . q_{n}\right) \text { for all } n=0,1,2, \cdots,
$$




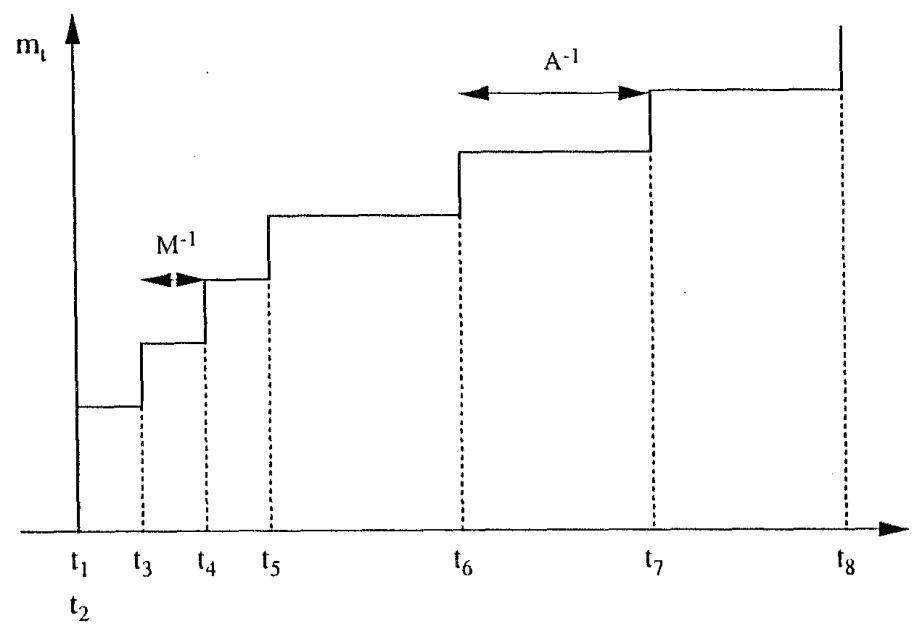

Fig. 6. Discrete time axis (for $C D V T>0$ ).

where

$$
\begin{aligned}
& p_{n} \stackrel{\text { def }}{=} p_{t_{n}}=\left\{\frac{A}{M}-\frac{A}{n}\left(\frac{1}{M}+T_{c}\right)\right\} \vee\left\{1-\frac{1}{n}\left(1+A T_{b}\right)\right\} \vee 0, \\
& q_{n} \stackrel{\text { def }}{=} q_{t_{n}}=\left\{\frac{c}{M}-\frac{1}{n}\left(\frac{c}{M}+c T_{c}-\kappa\right)\right\} \vee\left\{\frac{c}{A}-\frac{1}{n}\left(\frac{c}{A}+c T_{b}-\kappa\right)\right\} \vee \frac{\kappa}{n} .
\end{aligned}
$$

Remark: $\Psi_{N}^{G C R A}$ is not always the worst process of the original problem; the worst process of the original problem $\Psi_{T}^{G C R A}$ is not in $\left\{\Psi_{t_{n}}^{G C R A}\right\}_{n=1}^{\infty}$ if $T$ is not in the set $\left\{t_{n}\right\}_{n=1}^{\infty}$. We can determine, however, the lower and upper bounds of the rate function $I_{T}\left(\kappa+c T \mid \Psi_{T}^{G C R A}\right)$ for any $T$. In fact, for $t \in\left(t_{n}, t_{n+1}\right)$,

$$
\begin{aligned}
& p_{n} \leq p_{t} \leq \hat{p}_{n} \stackrel{\text { def }}{=}\left\{\frac{A}{M}-\frac{A T_{c}}{n}\right\} \vee\left\{1-\frac{A T_{b}}{n}\right\} \vee 0 \\
& q_{n} \leq q_{t} \leq \hat{q}_{n} \stackrel{\text { def }}{=}\left\{\frac{c}{M}-\frac{c T_{c}-\kappa}{n}\right\} \vee\left\{\frac{c}{A}-\frac{c T_{b}-\kappa}{n}\right\} \vee \frac{\kappa}{n}
\end{aligned}
$$

It thus follows from Lemma 3.3 that for $T \in\left(t_{n}, t_{n+1}\right)$,

$$
f\left(\hat{p}_{n}, q_{n}\right) \leq I_{T}\left(\kappa+c T \mid \Psi_{T}^{G C R A}\right)=f\left(p_{T}, q_{T}\right) \leq f\left(p_{n}, \hat{q}_{n}\right) .
$$

This enables us to estimate the worst case performance of a multiplexer based only on the results on the discrete time axis $\left\{t_{n}\right\}_{n=1}^{\infty}$.

\section{Case study}

4.1 Case 1: $C D V T=0$ and $B T=\infty$

First, we examine the simplest case where $T_{c}=0$ and $T_{b}=\infty$. The condition $T_{b}=\infty$ means that a cell-arrival process is not policed at the second-stage GCRA. (The average cell rate of an individual cell stream is, however, assumed to be bounded by $A$.) Under this condition,

$$
p_{n}=\frac{A}{M}-\frac{A}{n M}, \quad q_{n}=\frac{c}{M}-\frac{1}{n}\left(\frac{c}{M}-\kappa\right) .
$$

A: When $\kappa \geq c / M, p_{n}$ is increasing and $q_{n}$ is decreasing for all $n \geq 1$. In addition, $p_{n}<q_{n}$ for all $n \geq 1$ by the stability condition of the queue. Thus, we can apply Lemma 
3.3 for $q_{n}<1$ to obtain

$$
f\left(p_{n}, q_{n}\right) \geq f\left(p_{\infty}, q_{\infty}\right)=f\left(\frac{A}{M}, \frac{c}{M}\right)
$$

For $q_{n} \geq 1, f\left(p_{n}, q_{n}\right)=\infty$, so the above is satisfied for all $n \geq 1$. Thus, it follows from Lemmas 3.1 and 3.2 that $\Psi_{\infty}^{G C R A}$ is the worst in the sense of large-deviation ordering, and

$$
\inf _{t>0} I_{t}(\kappa+c t \mid \phi) \geq \inf _{t>0} I_{t}\left(\kappa+c t \mid \Psi_{\infty}^{G C R A}\right)=f\left(\frac{A}{M}, \frac{c}{M}\right) .
$$

Remark: Eq. (2.7) gives

$$
\begin{aligned}
P\left[Q^{L} \geq L \kappa\right] & \gtrsim \frac{1}{\sqrt{2 \pi L \theta_{\infty}^{*} \Lambda^{\prime \prime}\left(\theta_{\infty}^{*}\right)}} \exp \left\{-L f\left(\frac{A}{M}, \frac{c}{M}\right)\right\} \\
& =\frac{1}{\sqrt{2 \pi L \theta_{\infty}^{*}{ }^{2} \Lambda^{\prime \prime}\left(\theta_{\infty}^{*}\right)}} \exp \left\{-L\left(\frac{c}{M} \log \frac{c}{A}+\left(1-\frac{c}{M}\right) \log \frac{1-c / M}{1-A / M}\right)\right\}
\end{aligned}
$$

where

$$
\begin{aligned}
\theta_{\infty}^{* 2} \Lambda^{\prime \prime}\left(\theta_{\infty}^{*}\right) & =\lim _{t \rightarrow \infty} \theta_{t}^{* 2} A t\left(m_{t}^{G C R A}-A t\right) \\
& =\frac{A}{M}\left(1-\frac{A}{M}\right)\left(\log \frac{c}{A}-\log \frac{1-c / M}{1-A / M}\right)^{2}
\end{aligned}
$$

Observe that Stirling's formula gives for large $L$

$$
\begin{aligned}
& \log \left\{\left(\begin{array}{c}
L \\
c L / M
\end{array}\right)\left(\frac{A}{M}\right)^{c L / M}\left(1-\frac{A}{M}\right)^{L-c L / M}\right\} \\
= & -\log \sqrt{2 \pi L}-\log \sqrt{\frac{c}{M}\left(1-\frac{c}{M}\right)}-L \frac{c}{M} \log \frac{c}{A}-L\left(1-\frac{c}{M}\right) \log \frac{1-c / M}{1-A / M} \\
\simeq & -\log \sqrt{2 \pi L}-L\left(\frac{c}{M} \log \frac{c}{A}+\left(1-\frac{c}{M}\right) \log \frac{1-c / M}{1-A / M}\right) .
\end{aligned}
$$

We therefore approximately have for large $L$

$$
\begin{aligned}
C L R & \simeq\left(\begin{array}{c}
L \\
c L / M
\end{array}\right)\left(\frac{A}{M}\right)^{c L / M}\left(1-\frac{A}{M}\right)^{L-c L / M} \\
& \simeq \sum_{l: l M>c L}^{L}\left(\begin{array}{c}
L \\
l
\end{array}\right)\left(\frac{A}{M}\right)^{l}\left(1-\frac{A}{M}\right)^{L-l}
\end{aligned}
$$

The final exression is the same as the CLR of the bufferless model $[8,12,19]$, which is widely believed to be the worst CLR when $B T$ is infinite.

B: When $\kappa<c / M(<1), \Psi_{\infty}^{G C R A}$ is not always the worst because $p_{n}$ and $q_{n}$ are both increasing for all $n \geq 1$. In other words, the bufferless model may not give the worst performance when the buffer size per connection is smaller than $c / M$. A similar conclusion was reached by Saito [18] from a different approach.

Remark: Even in this case, we can obtain the lower bound of the rate function of the worst cell-arrival process. We define

$$
n_{0} \stackrel{\text { def }}{=} \min \left\{n \mid q_{n} \geq A / M=p_{\infty}\right\}=\left\lfloor\frac{c-\kappa M}{c-A}\right\rfloor .
$$


Because $p_{n} \leq p_{\infty} \leq q_{n_{0}} \leq q_{n}$ for $n \geq n_{0}$, we have

$$
\min _{n \geq 1} f\left(p_{n}, q_{n}\right)=\min _{n<n_{0}} f\left(p_{n}, q_{n}\right) \wedge \min _{n \geq n_{0}} f\left(p_{n}, q_{n}\right) \geq \min _{n<n_{0}} f\left(p_{n}, q_{n}\right) \wedge f\left(p_{\infty}, q_{n_{0}}\right) .
$$

Because $q_{n_{0}} \geq \kappa / n_{0}$, we finally have

$$
\begin{aligned}
\min _{n \geq 1} f\left(p_{n}, q_{n}\right) & \geq \min _{n<n_{0}} f\left(p_{n}, q_{n}\right) \wedge f\left(\frac{A}{M}, \frac{\kappa}{n_{0}}\right) \\
& =\min _{n<n_{0}} f\left(p_{n}, q_{n}\right) \wedge \frac{\kappa}{n_{0}} \log \frac{\kappa M}{n_{0} A}+\left(1-\frac{\kappa}{n_{0}}\right) \log \frac{1-\kappa / n_{0}}{1-A / M} .
\end{aligned}
$$

4.2 Case 2: $C D V T>0$ and $B T=\infty$

Next, we examine the case where $T_{c}>0$ and $T_{b}=\infty$, under which we have

$$
p_{n}=\left\{\frac{A}{M}-\frac{A}{n}\left(\frac{1}{M}+T_{c}\right)\right\} \vee 0, \quad q_{n}=\left\{\frac{c}{M}-\frac{1}{n}\left(\frac{c}{M}+c T_{c}-\kappa\right)\right\} \vee \frac{\kappa}{n} .
$$

The following discussion is analogous to that in Sec. 4.1.

A: When $\kappa \geq c / M+c T_{c}, p_{n}$ is an increasing and $q_{n}$ is a decreasing function for $n \geq 1$. It follows from Lemmas $3.1,3.2$, and 3.3 that $\Psi_{\infty}^{G C R A}$ is the worst in the sense of large-deviation ordering, and

$$
\inf _{t>0} I_{t}(\kappa+c t \mid \phi) \geq \inf _{t>0} I_{t}\left(\kappa+c t \mid \Psi_{\infty}^{G C R A}\right)=f\left(\frac{A}{M}, \frac{c}{M}\right) .
$$

This result points out a non-intuitive interesting fact: When $\kappa \geq c / M+c T_{c}$, the worst process is given by $\Psi_{\infty}^{G C R A}$, whose rate function $f\left(\frac{A}{M}, \frac{c}{M}\right)$ does not depend on $T_{c}$. That is, under the condition $\kappa \geq c / M+c T_{c}$ (i.e., we have a large buffer), the CDVT is negligible for the worst-case performance.

B: When $\kappa<c / M+c T_{c}$, we can obtain the lower bound of the rate function even though we cannot definitely identify the worst cell-arrival process. Observe that $p_{n}=0$ and $q_{n}=\kappa / n$ for $n<n_{1}$, and $p_{n}$ and $q_{n}$ are both increasing for $n \geq n_{1}$, where

$$
n_{1} \stackrel{\text { def }}{=}\left\lceil 1+T_{c} M\right\rceil \text {. }
$$

We define

$$
n_{0}=\min \left\{n \mid q_{n} \geq A / M=p_{\infty}\right\}=\left\lceil\frac{c\left(1+T_{c} M\right)-\kappa M}{c-A}\right\rceil .
$$

If $n_{0} \leq n_{1}$ (i.e., $\left.\kappa>A\left(1+T_{c} M\right) / M\right)$, we have

$$
\begin{aligned}
\min _{n \geq 1} f\left(p_{n}, q_{n}\right) & \geq \min _{1 \leq n<n_{1}} f\left(p_{n}, q_{n}\right) \wedge \min _{n_{1} \leq n} f\left(p_{n}, q_{n}\right) \\
& \geq f\left(0, \kappa /\left(n_{1}-1\right)\right) \wedge f\left(p_{\infty}, q_{n_{1}}\right)=f\left(\frac{A}{M}, q_{n_{1}}\right),
\end{aligned}
$$

where the last equality is derived from the fact $f\left(0, \kappa /\left(n_{1}-1\right)\right)=\infty$. Because $q_{n_{1}} \geq \kappa / n_{1}$, we have

$$
\min _{n \geq 1} f\left(p_{n}, q_{n}\right) \geq f\left(\frac{A}{M}, \kappa / n_{1}\right)=\frac{\kappa}{n_{1}} \log \frac{\kappa M}{n_{1} A}+\left(1-\frac{\kappa}{n_{1}}\right) \log \frac{1-\kappa / n_{1}}{1-A / M} .
$$

If $n_{1}<n_{0}$, we obtain

$$
\begin{aligned}
\min _{n \geq 1} f\left(p_{n}, q_{n}\right) & \geq \min _{1 \leq n<n_{1}} f\left(p_{n}, q_{n}\right) \wedge \min _{n_{1} \leq n<n_{0}} f\left(p_{n}, q_{n}\right) \wedge \min _{n_{0} \leq n} f\left(p_{n}, q_{n}\right) \\
& \geq f\left(0, \kappa /\left(n_{1}-1\right)\right) \wedge \min _{n_{1} \leq n<n_{0}} f\left(p_{n}, q_{n}\right) \wedge f\left(p_{\infty}, q_{n_{0}}\right) \\
& \geq \min _{n_{1} \leq n_{0}<n_{0}} f\left(p_{n}, q_{n}\right) \wedge f\left(\frac{A}{M}, \frac{\kappa}{n_{0}}\right) .
\end{aligned}
$$


4.3 Case 3: $C D V T=0$ and $B T<\infty$

Finally, we examine the case where $T_{c}=0$ and $T_{b}<\infty$. Although this case has been extensively studied $[8,17,22,23]$, no conclusive answer has been reached. Under the condition considered here,

$$
\begin{aligned}
& p_{n}=\left(\frac{A}{M}-\frac{A}{n M}\right) \vee\left\{1-\frac{1}{n}\left(1+A T_{b}\right)\right\} \\
& q_{n}=\left\{\frac{c}{M}-\frac{1}{n}\left(\frac{c}{M}-\kappa\right)\right\} \vee\left\{\frac{c}{A}-\frac{1}{n}\left(\frac{c}{A}+c T_{b}-\kappa\right)\right\} .
\end{aligned}
$$

Note that $p_{n}$ is increasing for all $n \geq 1$.

A: When $c / M<c / A+c T_{b} \leq \kappa, q_{n} \geq 1$ because $q_{n}$ is decreasing for $n$ and $q_{\infty}=1$. Thus, $f\left(p_{n}, q_{n}\right)=\infty$ for all $n \geq 1$. In fact,

$$
m_{t}^{G C R A} \leq 1+\left(t+T_{b}\right) A \leq \frac{A}{c}(\kappa+c t)<\kappa+c t
$$

which implies no cells are lost.

B: When $c / M \leq \kappa<c / A+c T_{b}, q_{n}$ is decreasing for $1 \leq n \leq n_{2}$ and increasing for $n>n_{2}$, where

$$
n_{2} \stackrel{\text { def }}{=} \max \left\{n \mid \frac{c}{M}-\frac{1}{n}\left(\frac{c}{M}-\kappa\right) \geq \frac{c}{A}-\frac{1}{n}\left(\frac{c}{A}+c T_{b}-\kappa\right)\right\}=1+\left\lfloor\frac{M A T_{b}}{M-A}\right\rfloor .
$$

Thus, it follows from Lemma 3.2 that

$$
f\left(p_{n}, q_{n}\right) \geq f\left(p_{n_{2}}, q_{n_{2}}\right) \wedge \min _{n>n_{2}} f\left(p_{n}, q_{n}\right) .
$$

Note that $n_{2}$ is the maximum number of cells sent in a "burst" at peak rate $M$; this is usually referred to as the maximum burst size (MBS) [2]. If we have

$$
\min _{n>n_{2}} f\left(p_{n}, q_{n}\right) \geq f\left(p_{n_{2}}, q_{n_{2}}\right)
$$

$\Psi_{n_{2}}^{G C R A}$ is the worst in the sense of large-deviation ordering. The number of cells from $\Psi_{n_{2}}^{G C R A}$ in any interval of length $t_{n_{2}}=n_{2} / M$ is equal to the $\operatorname{MBS}\left(=n_{2}\right)$ or 0 , so in this sense $\Psi_{n_{2}}^{G C R A}$ corresponds to the greedy on-off source. Conversely, if we have

$$
f\left(p_{n_{2}}, q_{n_{2}}\right) \geq \min _{n>n_{2}} f\left(p_{n}, q_{n}\right)=f\left(p_{n_{3}}, q_{n_{3}}\right) \quad \text { for some } \quad n_{3}>n_{2},
$$

$\Psi_{n_{3}}^{G C R A}$ is the worst in the sense of large-deviation ordering. Because cell-arrival process $\Psi_{n_{3}}^{G C R A}$ is not the greedy on-off pattern, this supports Yamanaka's conjecture. In the following, the derivative of $f\left(p_{n}, q_{n}\right)$ by $n$ for $n>n_{2}$ is further calculated. First observe that for $n>n_{2}$

$$
p_{n}=1-\frac{1}{n}\left(1+A T_{b}\right), \quad \text { and } \quad q_{n}=\frac{c}{A}-\frac{1}{n}\left(\frac{c}{A}+c T_{b}-\kappa\right) .
$$

Thus,

$$
\begin{aligned}
\Delta f\left(p_{n}, q_{n}\right) & =\Delta p_{n} \frac{d}{d p_{n}} f\left(p_{n}, q_{n}\right)+\Delta q_{n} \frac{d}{d q_{n}} f\left(p_{n}, q_{n}\right) \\
& =\log \frac{q_{n}\left(1-p_{n}\right)}{p_{n}\left(1-q_{n}\right)} \Delta q_{n}-\frac{q_{n}-p_{n}}{p_{n}\left(1-p_{n}\right)} \Delta p_{n} \\
& =\frac{1}{n+1}\left\{\left(\frac{c}{A}-q_{n}\right) \log \frac{q_{n}\left(1-p_{n}\right)}{p_{n}\left(1-q_{n}\right)}-\frac{q_{n}-p_{n}}{p_{n}}\right\},
\end{aligned}
$$


where

$$
\Delta f(n) \stackrel{\text { def }}{=} f(n+1)-f(n)
$$

Further, consider the following Taylor expansion:

$$
\begin{aligned}
\log \frac{q_{n}\left(1-p_{n}\right)}{p_{n}\left(1-q_{n}\right)} & =\log \left(1+\frac{q_{n}-p_{n}}{p_{n}\left(1-q_{n}\right)}\right) \\
& =\frac{q_{n}-p_{n}}{p_{n}\left(1-q_{n}\right)}-\frac{1}{2}\left(\frac{q_{n}-p_{n}}{p_{n}\left(1-q_{n}\right)}\right)^{2}+\frac{1}{3}\left(\frac{q_{n}-p_{n}}{p_{n}\left(1-q_{n}\right)}\right)^{3}+\cdots
\end{aligned}
$$

It is used to obtain

$$
\begin{aligned}
\Delta f\left(p_{n}, q_{n}\right) & =\frac{q_{n}-p_{n}}{(n+1) p_{n}}\left[-1+\frac{c / A-q_{n}}{1-q_{n}}\left\{1-\frac{1}{2} g\left(p_{n}, q_{n}\right)+\frac{1}{3} g\left(p_{n}, q_{n}\right)^{2}+\cdots\right\}\right] \\
g\left(p_{n}, q_{n}\right) & \stackrel{\text { def }}{=} \frac{q_{n}-p_{n}}{p_{n}\left(1-q_{n}\right)}
\end{aligned}
$$

If the second and higher-order terms of the expansion on the right side of Eq. (4.28) are neglected, we obtain

$$
\Delta f\left(p_{n}, q_{n}\right) \simeq \frac{q_{n}-p_{n}}{n p_{n}}\left[-1+\frac{c / A-q_{n}}{1-q_{n}}\right]>0,
$$

and thus

$$
f\left(p_{n}, q_{n}\right) \geq f\left(p_{n_{2}}, q_{n_{2}}\right) \wedge f\left(p_{n_{2}+1}, q_{n_{2}+1}\right) \simeq f\left(p_{n_{2}}, q_{n_{2}}\right) .
$$

(In fact, $g\left(p_{n}, q_{n}\right)=o\left(n^{-1}\right)$, and thus, for large $n, g\left(p_{n}, q_{n}\right) \ll 1$.) This suggests that the general belief - that the greedy on-off pattern is the worst-is correct. We do not, however, obtain that conclusion if we consider the higher-order terms on the right side of Eq. (4.28). In particular, when $A \simeq c$, the greedy on-off source is not the worst pattern, as we could anticipate from Eq. (4.28).

C: When $\kappa<c / M<c / A+c T_{b}, p_{n}$ and $q_{n}$ are both increasing for all $n \geq 1$. In this case, we have

$$
\min _{n \geq 1} f\left(p_{n}, q_{n}\right)=\min _{1 \leq n<n_{0}} f\left(p_{n}, q_{n}\right) \wedge \min _{n \geq n_{0}} f\left(p_{n}, q_{n}\right) \geq \min _{1 \leq n<n_{0}} f\left(p_{n}, q_{n}\right)
$$

where

$$
n_{0} \stackrel{\text { def }}{=} \min \left\{n \mid q_{n} \geq 1=p_{\infty}\right\}=1+\left\lceil\frac{A}{c}\left(c T_{b}-\kappa\right)\right\rceil
$$

\section{Numerical examples}

For the cell-arrival processes conforming to dual GCRAs, we numerically found the set of parameter values under which the greedy on-off pattern becomes the worst in the sense of large-deviation ordering. The same condition as Case 3 in Sec. 4 was assumed that is, the cell-delay variation tolerance of each cell-arrival process was 0 . The capacity of the outgoing link per connection $c$ was assumed to be $2 \mathrm{Mbps}$.

Figures 7(a)-(d) illustrate the region where the greedy on-off pattern is the worst on the PCR-SCR map for four different buffer sizes per connection. The burst tolerance was set to $1 \mathrm{~ms}$. These figures show that

1. Regions where the greedy on-off pattern is not the worst exist and, in this sense, Yamanaka's conjecture is confirmed. 


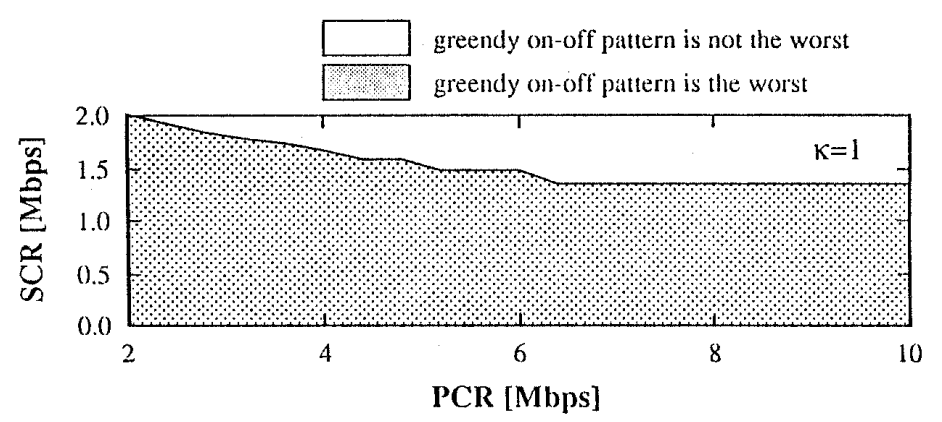

(a)

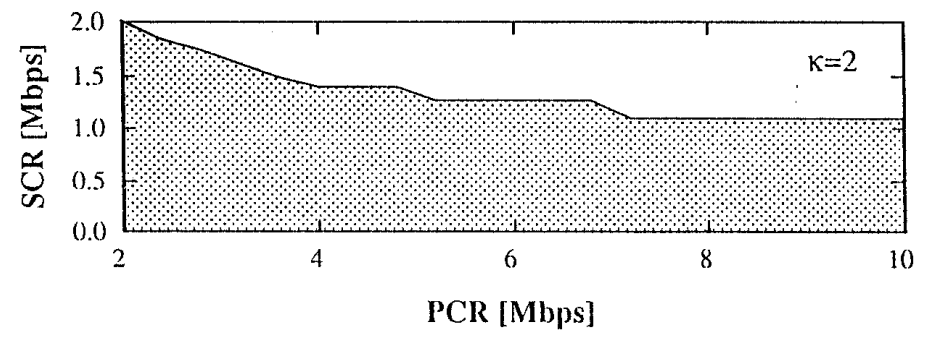

(b)

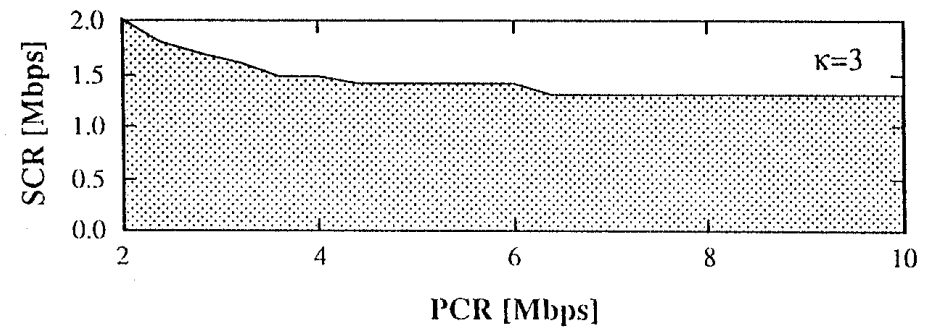

(c)

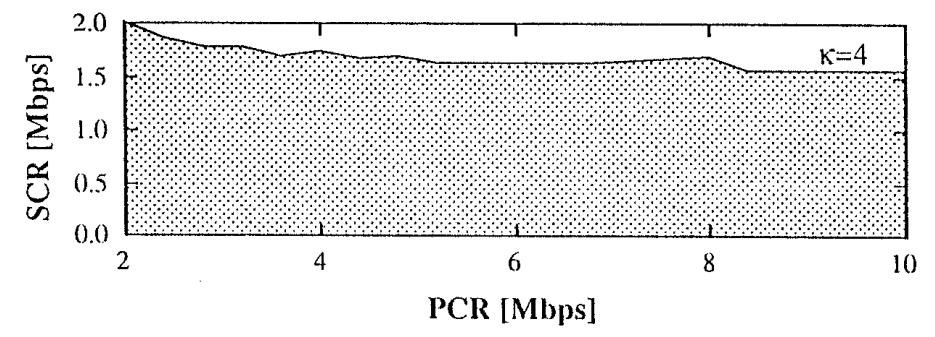

(d)

Fig. 7. Region where greedy on-off source is the worst when $B T=1 \mathrm{~ms}$.

2. When $S C R \ll c$, the greedy on-off source is the worst regardless of the PCR and of the buffer size per connection. In contrast, when $S C R \simeq c$, the greedy on-off pattern is not the worst.

3. When $P C R \simeq c$, the greedy on-off source is the worst regardless of the SCR and of the buffer size per connection. As the PCR becomes larger (compared with $c$ ), however, the region where the greedy on-off pattern is the worst becomes smaller.

Ethernet LAN traffic is quite bursty and thus its average rate is much smaller than the capacity assigned to the connection. Therefore, for connections dedicated to such traffic, we can regard the greedy on-off source as the worst. For connections dedicated to voice or video traffic, however, resource assignment based on the assumption that the greedy on-off source is the worst might lead to QoS degradation.

Figures $8(\mathrm{a})-(\mathrm{d})$ illustrate the region where the greedy on-off pattern is the worst on the PCR-SCR map when the burst tolerance is set to $10 \mathrm{~ms}$. The same tendencies are observed 


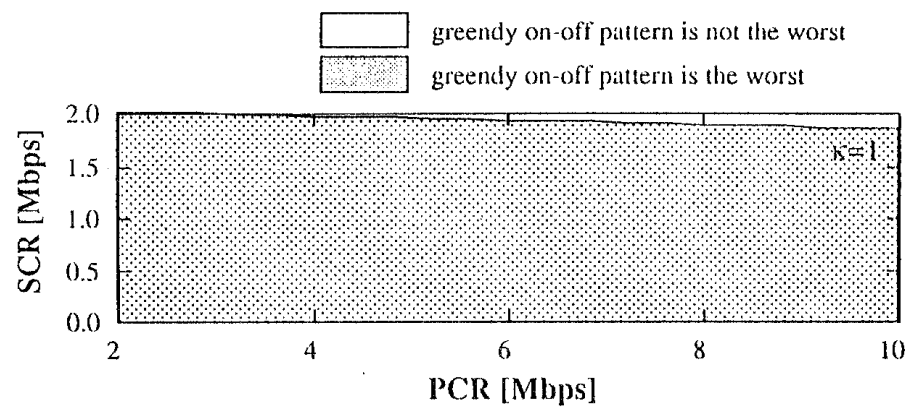

(a)

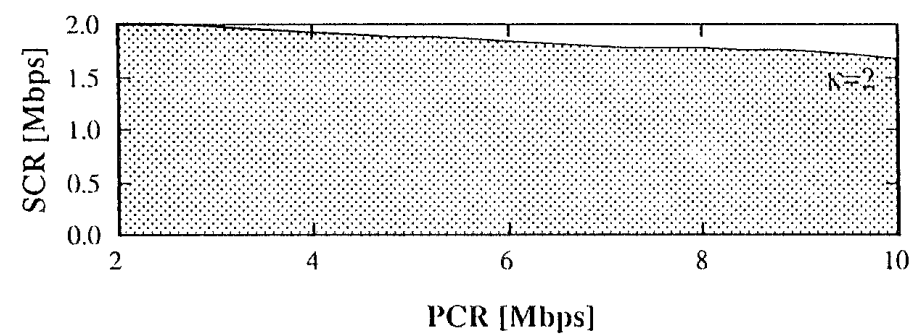

(b)

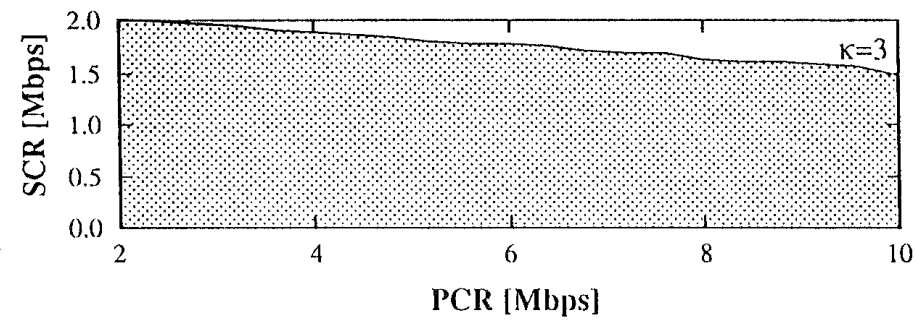

(c)

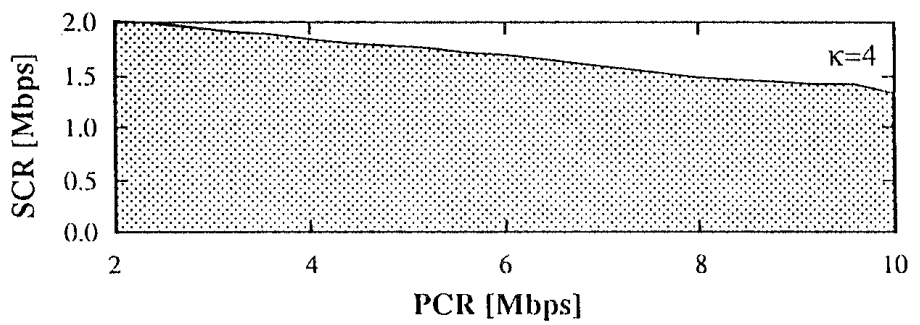

(d)

Fig. 8. Region where greedy on-off source is the worst when $B T=10 \mathrm{~ms}$.

as in Figs. 7(a)-(d). Furthermore, compared with Figs. 7(a)-(d), Figs. 8(a)-(d) show that as the burst tolerance becomes larger, the regions where the greedy on-off pattern is the worst become wider (except for the case shown in Fig. 8(d)).

\section{Conclusion}

We have examined the worst-case performance of an ATM multiplexer, in which each multiplexed connection sends cells so as to confirm to the generic cell rate algorithm. Specifically, we examined the case where a large number of connections share a multiplexer and discussed the worst cell-arrival process based on the concept of large-deviation ordering. We obtained several interesting results. First, the cell loss ratio of the bufferless model is not always the worst cell loss ratio in the multiplexer when the buffer size per connection is less than $c / M$, where $c$ is the outgoing-link capacity per connection and $M$ is the peak rate of the connection. Second, the cell-delay-variation tolerance does not affect the worst-case performance if the buffer size is sufficiently large. The implication of this is very useful 
because it can simplify the worst-case-resource assignment, which is the most promising strategy for resource assignment in ATM networks. Finally, in the sense of large-deviation ordering, we derived theoretical support for Yamanaka's conjecture that the greedy on-off pattern is not always the worst.

Compared with previous studies, this paper's approach has the major advantage of simplicity: in this framework, the worst-case performance is given by solving an optimization problem, such as minimizing the rate function. This approach is therefore very easy to handle and is expected to provide fruitful insights into the worst case.

Finally, we discuss a possible extension of these results to the heterogeneous environment. The heterogeneous version of Eq. (1.2) is given as follows: assume that there are $L_{j}$ connections of type $j$, with $L=\sum_{j} L_{j}$ connections in total. The arrival process from a type $j$ connection is denoted as $A_{j, t}$. Therefore,

$$
\lim _{L \rightarrow \infty} \frac{1}{L} \log \mathbf{P}\left[Q^{L} \geq L \kappa\right]=-\inf _{t>0}\left\{\sup _{\theta}\left[\theta(\kappa+c t)-\sum_{j} r_{j} \log E\left[e^{\theta A_{j, t}}\right]\right]\right\}
$$

where

$$
r_{j}=\lim _{L \rightarrow \infty} L_{j} / L
$$

Equation (6.1) is still simple enough to analytically derive results for worst-case performance in a heterogeneous environment. Furthermore, Eq. (6.1) indicate that the worst cell-arrival process may depend on the source $\operatorname{mix}\left\{r_{j}\right\}$, which might make the resource allocation in the heterogeneous environment more complex.

Acknowledgments We would like to thank the referees for their very careful review of this paper. Their insightful comments have led to a greatly improved verison of the paper.

\section{References}

[1] Draft Recommendation I.371: Traffic congestion control in B-ISDN (1994).

[2] M. Aida: Congestion detection and CAC for ABR services using allan variance, IEICE Trans. Commun., E79-B(4) (1996) 540-549.

[3] F. Brichet, L. Massoulié, and J. W. Roberts: Stochastic ordering and the notion of negligible CDV, ITC 15 (1997) 1433-1444.

[4] D. Botvich and N. Duffield: Large deviations, the shape of the loss curve, and economies of scale in large multiplexers, Queueing Systems, 20 (1995) 293-320.

[5] C. S. Chang: Stability, queue length, and delay of deterministic and stochastic queueing networks, IEEE Trans. Auto. Cont., 39(5) (1994) 913-931.

[6] C. Courcoubetis, G. Fouskas, and R. Weber: On the performance of an effective bandwidth formula, ITC 14 (1994) 191-200.

[7] A. Dembo and O. Zeitouni: Large Deviations Techniques and Applications (Jones and Bartlett Publishers, 1993).

[8] B. T. Doshi: Deterministic rule based traffic descriptors for broadband ISDN: worst case behavior and connection acceptance control, ITC 14 (1994) 591-600.

[9] N. G. Duffield: Exponential bounds for queues with Markovian arrivals, Queueing Systems, 17 (1994) 413-430.

[10] N. G. Duffield, J. T. Lewis, N. O'Connell, R. Russel, and F. Toomey: Entropy of ATM traffic streams: A tool for estimating QoS parameters, IEEE J. S. A. C., 13(6) (1995) 981-990. 
[11] A. Elwalid, D. Mitra, and R. Wentworth: A new approach for allocating buffers and bandwidth to heterogeneous, regulated traffic in an ATM node, IEEE J. S. A. C., 13(6) (1995) 1115-1127.

[12] A. Elwalid, D. Heyman, T. V. Lakshman, D. Mitra, and A. Weiss: Fundamental bounds and approximations for ATM multiplexers with applications to video teleconferencing, IEEE J. S. A. C., 13(6) (1995) 1004-1016.

[13] P. Glynn and W. Whitt: Large deviations behavior of counting processes and their inverses, Queueing Systems, 17 (1994) 107-128.

[14] A. Gravey: Cell conformance and quality of service guarantees in ATM networks, ITC 14 (1994) 1395-1404.

[15] I. Hsu and J. Walrand: Admission control for ATM networks. In F. P. Kelly and R. J. Williams (eds.): Stochastic Networks (The IMA Volume, Springer-Verlag, 1995), 411-427.

[16] F. P. Kelly: Effective bandwidth at multi-class queues, Queueing Systems, 9 (1991) 5-16.

[17] D. C. Lee: Effects of leaky bucket parameters on the average queue delay: worst case analysis, INFOCOM'94 (1994) 482-489.

[18] H. Saito: Toward a future traffic dimensioning method: non-parametric approach for cell loss rate evaluation, IEICE Trans. Commun., J76-B-I(3) (1993) 197-208.

[19] F. C. Schoute: Simple decision rules for acceptance of mixed traffic streams, Philips TDS Reviews, 46(2) (1988) 351-358.

[20] S. Shioda and H. Saito: A connection admission control guaranteeing a negotiated cellloss ratio of the cell streams passing through usage parameter control, IEICE Trans. Commun., E80-B(3) (1997) 399-411.

[21] D. Stoyan: Comparison Methods for Queues and Other Stochastic Models, (John Wiley, Berlin, 1983).

[22] T. Tsuchiya and H. Saito: The worst case cell arrival patterns that conform to the GCRA in ATM networks, GLOBECOM'95 (1995).

[23] N. Yamanaka, Y. Sato, and K. Sato: Performance limitation of the leaky bucket algorithm for ATM networks, IEEE Trans. Commun., 43(8) (1995) 2298-2230.

[24] W. Whitt: Tail probabilities with statistical multiplexing and effective bandwidth in multiclass queues, Telecommunication Systems, 2 (1993) 71-107.

Shigeo Shioda

NTT Tokyo District Headquarters

NTT Shinagawa TWINS 10F, 1-9-1 Kounan, Minato-ku, Tokyo 108-19, Japan

E-mail: shioda.shigeo@psd.tbn.ntt.co.jp 